\title{
Elucidating distinct clinico-radiologic signatures in the borderland between neuromyelitis optica and multiple sclerosis
}

\author{
Maciej Juryńczyk ${ }^{1,2}$ (1) Elżbieta Klimiec-Moskal ${ }^{1,3} \cdot$ Yazhuo Kong $^{4,5,6} \cdot$ Samuel Hurley $^{4} \cdot$ Silvia Messina $^{1}$. \\ Tianrong $\mathrm{Yeo}^{1} \cdot$ Mark Jenkinson $^{4} \cdot$ Maria Isabel Leite ${ }^{1} \cdot$ Jacqueline Palace $^{1}$
}

Received: 4 January 2021 / Revised: 14 May 2021 / Accepted: 18 May 2021 / Published online: 27 May 2021

(c) The Author(s) 2021

\begin{abstract}
Background Separating antibody-negative neuromyelitis optica spectrum disorders (NMOSD) from multiple sclerosis (MS) in borderline cases is extremely challenging due to lack of biomarkers. Elucidating different pathologies within the likely heterogenous antibody-negative NMOSD/MS overlap syndrome is, therefore, a major unmet need which would help avoid disability from inappropriate treatment.

Objective In this study we aimed to identify distinct subgroups within the antibody-negative NMOSD/MS overlap syndrome. Methods Twenty-five relapsing antibody-negative patients with NMOSD features underwent a prospective brain and spinal cord MRI. Subgroups were identified by an unsupervised algorithm based on pre-selected NMOSD/MS discriminators.

Results Four subgroups were identified. Patients from Group 1 termed "MS-like" $(n=6)$ often had central vein sign and cortical lesions (83\% and 67\%, respectively). All patients from Group 2 ("spinal MS-like", 8) had short-segment myelitis and no MS-like brain lesions. Group 3 ("classic NMO-like", 6) had high percentage of bilateral optic neuritis and longitudinally extensive transverse myelitis (LETM, 80\% and 60\%, respectively) and normal brain appearance (100\%). Group 4 ("NMO-like with brain involvement", 5) typically had a history of NMOSD-like brain lesions and LETM. When compared with other groups, Group 4 had significantly decreased fractional anisotropy in non-lesioned tracts $(0.46$ vs. $0.49, p=0.003)$ and decreased thalamus volume (0.84 vs. $0.98, p=0.04)$.

Conclusions NMOSD/MS cohort contains distinct subgroups likely corresponding to different pathologies and requiring tailored treatment. We propose that non-conventional MRI might help optimise diagnosis in these challenging patients.
\end{abstract}

Keywords Multiple sclerosis $\cdot$ Neuromyelitis optica $\cdot$ Optic neuritis $\cdot$ Myelitis $\cdot$ Magnetic resonance imaging $\cdot$ Prospective studies

\begin{tabular}{|c|c|c|c|}
\hline & & \multicolumn{2}{|c|}{ Abbreviations } \\
\hline & & $\mathrm{BON}$ & Bilateral optic neuritis \\
\hline & & \multicolumn{2}{|r|}{ Double inversion recovery } \\
\hline & & \multicolumn{2}{|r|}{ Diffusion tensor imaging } \\
\hline \multicolumn{2}{|r|}{$\begin{array}{l}\text { Maciej Juryńczyk and Elżbieta Klimiec-Moskal contributed } \\
\text { equally. }\end{array}$} & \multicolumn{2}{|r|}{ Fluid attenuated inversion recovery } \\
\hline$\triangle$ & $\begin{array}{l}\text { Maciej Juryńczyk } \\
\text { m.jurynczyk@ nencki.edu.pl } \\
\text { Jacqueline Palace }\end{array}$ & $\begin{array}{l}\text { Depa } \\
\text { Oxfo }\end{array}$ & $\begin{array}{l}\text { ne Centre for Integrative Neuroimaging, Nuffield } \\
\text { nent of Clinical Neurosciences, University of Oxford, } \\
\text { UK }\end{array}$ \\
\hline$\square$ & jacqueline.palace@ndnc.ox.ac.uk & \multirow{2}{*}{ of Ps } & $\begin{array}{l}\text { y Laboratory of Behavioral Science, Institute } \\
\text { ology, Chinese Academy of Sciences, }\end{array}$ \\
\hline \multirow{2}{*}{\multicolumn{2}{|c|}{$\begin{array}{l}1 \text { Department of Clinical Neurology, Nuffield Department } \\
\text { of Clinical Neuroscienes, University of Oxford, Oxford, UK }\end{array}$}} & & 100101, China \\
\hline & & Belj1 & nent of Psychology, University of Chinese Academy \\
\hline 2 & $\begin{array}{l}\text { Laboratory of Brain Imaging, Neurobiology Center, } \\
\text { Nencki Institute of Experimental Biology, Polish Academy } \\
\text { of Sciences, Warsaw, Poland }\end{array}$ & of $\mathrm{Sc}$ & ces, Beijing 100049, China \\
\hline 3 & Department of Neurology, Jagiellonian University Medical & & \\
\hline
\end{tabular}


LETM Longitudinally extensive transverse myelitis

MOG Myelin oligodendrocyte glycoprotein

NMOSD Neuromyelitis optica spectrum disorders

PCA Principal component analysis

\section{Introduction}

Since the nineteenth century neuromyelitis optica (NMO, Devic's syndrome) defined as acute simultaneous bilateral optic neuritis (BON) and transverse myelitis has been regarded as different from typical MS. The uniqueness of NMO has been emphasised by poor visual and motor outcome, and longitudinal extension of the transverse myelitis lesion (termed LETM) [1]. NMO was finally recognised as separate from MS when a subset of NMO patients were found to have serum antibodies against aquaporin-4 [2, 3]. This discovery broadened the spectrum of NMO (termed NMOSD) to include BON, isolated LETM and other limited forms of NMO [4].

Some patients with features of NMOSD have serum antibodies against MOG rather than aquaporin-4 [5, 6]. MOG-antibody disease (MOGAD) has similar brain imaging appearances to AQP4-antibody NMOSD, which are both easily distinguishable from MS [7]. In particular, AQP4antibody NMOSD and MOGAD patients rarely fulfil MS brain lesion distribution criteria $[8,9]$.

The recognition of aquaporin-4-antibody NMOSD and MOGAD as separate diseases sparked interest in the cohort of patients with NMOSD features and red flags for the diagnosis of MS who are negative for both autoantibodies. It is widely accepted that this cohort is highly heterogenous and likely contains distinct diagnoses ranging from atypical multiple sclerosis to NMOSD mediated by yet undiscovered antibodies. Making ultimate diagnosis is very challenging as shown by high disagreement between experts when presented with individual cases [10]. According to the revised McDonald criteria for the diagnosis of MS, NMOSD should be considered in all patients with NMOSD features such as BON, severe brainstem involvement, longitudinally extensive spinal cord lesions, large cerebral lesions, or normal brain MRI [11]. It is well recognised that AQP4-antibody NMOSD and MOGAD patients can formally meet McDonald criteria [12-14]. A degree of diagnostic uncertainty in seronegative patients also occurs when applying NMOSD diagnostic criteria [15]. The correct diagnosis is however essential since typical NMOSD drugs are not licensed in MS nor are the treatments of choice, and MS-modifying drugs might exacerbate the course of NMOSD [16, 17].

In this study we analysed clinical, paraclinical and non-conventional imaging features of 25 antibody-negative patients recruited from the Specialist NMO Clinic in Oxford with recurrent syndromes at the borderline of MS and NMOSD, to identify patient subgroups, propose classification and guide clinicians when diagnosing and treating these challenging patients.

\section{Methods}

\section{Patients}

Patient inclusion criteria included (1) the presence of at least one NMOSD feature as per 2007 criteria [4], (2) seronegativity for both aquaporin-4 and MOG antibodies, (3) recurrent disease course.

\section{MRI scan acquisition}

All participants underwent an MRI scan of the brain and cervical spinal cord in the Wellcome Centre for Integrative Neuroimaging in Oxford. The scan was obtained using a 3 T Siemens Prisma. The protocol sequences included T1 MP-RAGE, T2 FLAIR, T2 double inversion recovery (DIR), T2* mapping and diffusion tensor imaging (DTI) for the brain and T1 MP-RAGE and T2 FLAIR for the spinal cord.

\section{Image processing and analysis}

The identification of cortical lesions on DIR was based on the consensus between two experienced raters (MJ, EKM). The presence or absence of the central vein sign was assessed according to the guidelines [18]. FLAIR images were scored for the presence of MS brain distribution criteria $[8,9]$. Normalised brain volumes were obtained using FSL Sienax [19]. Subcortical structures and brainstem were segmented using FIRST and their volume was calculated using FSL command fslstats based on the label number of the structure of interest obtained from FIRST [20]. Cortical thickness was quantified using the Freesurfer software (version 6.0) [21]. To measure fractional anisotropy in nonlesioned tracts lesion masks were registered to T1, inverted and multiplied by masks of the white matter tracts of interest obtained from a DTI-based white matter atlas [22]. Freesurfer was implemented to obtain cortical ribbon masks, which were used when assessing mean diffusivity in the cortex. An R2*-weighted image was produced using a gradient echo MR sequence with a long echo time. A quantitative value for $\mathrm{R} 2 *$ was calculated in each voxel in units of [1/s].

The spinal cord was processed and automatically segmented using a deep learning algorithm implemented within the Spinal Cord Toolbox [23]. Vertebral disc labelling was performed manually and the cross-sectional area was calculated for each segment in the native space. 


\section{Selection of discriminators for patient subgroup identification}

Eleven NMO/MS discriminators were selected to separate patient subgroupings, including $\mathrm{BON}$, poor residual visual acuity after optic neuritis (6/36 or worse in at least one eye), CSF oligoclonal bands unmatched in the serum, LETM, short-segment transverse myelitis, NMO-like brain lesions (all from clinical files or clinical scans performed prior to the study recruitment), MRI MS brain lesion distribution criteria (at least 1 lesion adjacent to the body of the lateral ventricle and in the inferior temporal lobe; or the presence of a subcortical U-fibre lesion; or a Dawson's finger-type lesion) [8], cortical lesions, central vein sign, thalamus volume, fractional anisotropy in non-lesioned white matter tracts (as assessed on the prospective research images). Fractional anisotropy was measured in all non-lesioned tracts, apart from the optic radiation to exclude the potential effect of previous optic neuritis.

\section{Unsupervised identification of patient subgroups}

PCA on the obtained data matrix was performed using the prcomp function within the $\mathrm{R}$ statistical software. Visualisation of the first two principal components indicated the existence of four separate patient subgroups. To objectively determine which patients form each subgroup, $k$-means clustering (with four centres) was applied using $k$-means function from R software. As PCA normalises the data and uncorrelates the variables, the clustering was performed on the PCA matrix rather than original data. A scree plot was generated to show the proportion of variance explained by each principal component and to decide the number of principal components included in the matrix (the first four components, cumulatively explaining $69 \%$ of the variance, were ultimately used). Figure 1 shows the flow chart of the study.

\section{Quantitative and non-conventional imaging measures not selected as a priori discriminators}

After subgroup identification various non-conventional imaging measures reflecting different aspects of tissue damage were analysed in the four identified subgroups: fractional anisotropy in corticospinal tracts, corpus callosum and optic radiation, $\mathrm{R} 2 *$ relaxometry in the normal-appearing white matter, normalised brain volume, normalised volume of basal ganglia (caudate, pallidum and putamen combined), mean diffusivity in the cortex, cortical thickness and mean cervical cord cross-sectional area). We selected fractional anisotropy rather than mean diffusivity as a measure of structural damage in the normal-appearing white matter $[24,25]$. Mean diffusivity was used to assess damage in the cortex [26].

\section{Results}

\section{Patients}

Twenty-five patients were included in the study of whom 15 were female. Mean age at scan was 49 years (range 20-73) and median disease duration was 9.8 years (range 1-28). All patients were relapsing, their median number of attacks was three (range 2-11) and median EDMUS was three (range 0-8). Twelve patients had previous attacks of LETM, five had had BON and six were left with visual acuity at 6/36 or worse in at least one eye. Five patients were reported to have NMO-like brain lesions at some stage of their disease. Thirteen patients had CSF-exclusive oligoclonal bands. At the time of the research scan nine patients were not on any disease-modifying treatment, six were on azathioprine, five on methotrexate, two on mycophenolate mofetil, one on prednisolone alone, one on regular intravenous immunoglobulins and one on fingolimod. The breakdown of NMOand MS-like features for individual patients is shown in Supplementary Table S1.

\section{Overview of brain lesions on the research scans}

Eight out of 25 patients had distinct white matter lesions in the brain on FLAIR-weighted images (four had one lesion, one had two lesions, one had three lesions and two had four lesions). Five patients had Dawson's finger(s), three had lesions adjacent to the body of lateral ventricle, two had infratentorial temporal lobe lesions and one had curved juxtacortical lesions. Seven patients fulfilled MS brain lesion distribution criteria [8]. Six had at least one lesion centred by a vein but only four fulfilled the ' $40 \%$ rule' [27]. Five patients had at least one cortical lesion on FLAIR/DIR images.

\section{Patient subgroups}

Four separate subgroups were identified (Fig. 2). Group 1 was characterised by a high percentage of positivity for brain lesion distribution criteria $(83 \%)$, central vein sign $(83 \%)$ and cortical lesions (67\%, Table 1). Group 2 consisted of patients who all had short-segment transverse myelitis but did not have MS brain lesions according to the criteria. Group 3 had a high percentage of BON and LETM $(80 \%$ and $60 \%$, respectively) but did not have any previous or current brain lesions (100\%). Group 4 also consisted of LETM patients $(100 \%)$ who, however, also typically had a history of NMOSD-like brain lesions (67\%).

When compared with other groups, patients from Group 4 had significantly decreased fractional anisotropy 


\section{5 antibody-negative MS/NMOSD overlap patients}

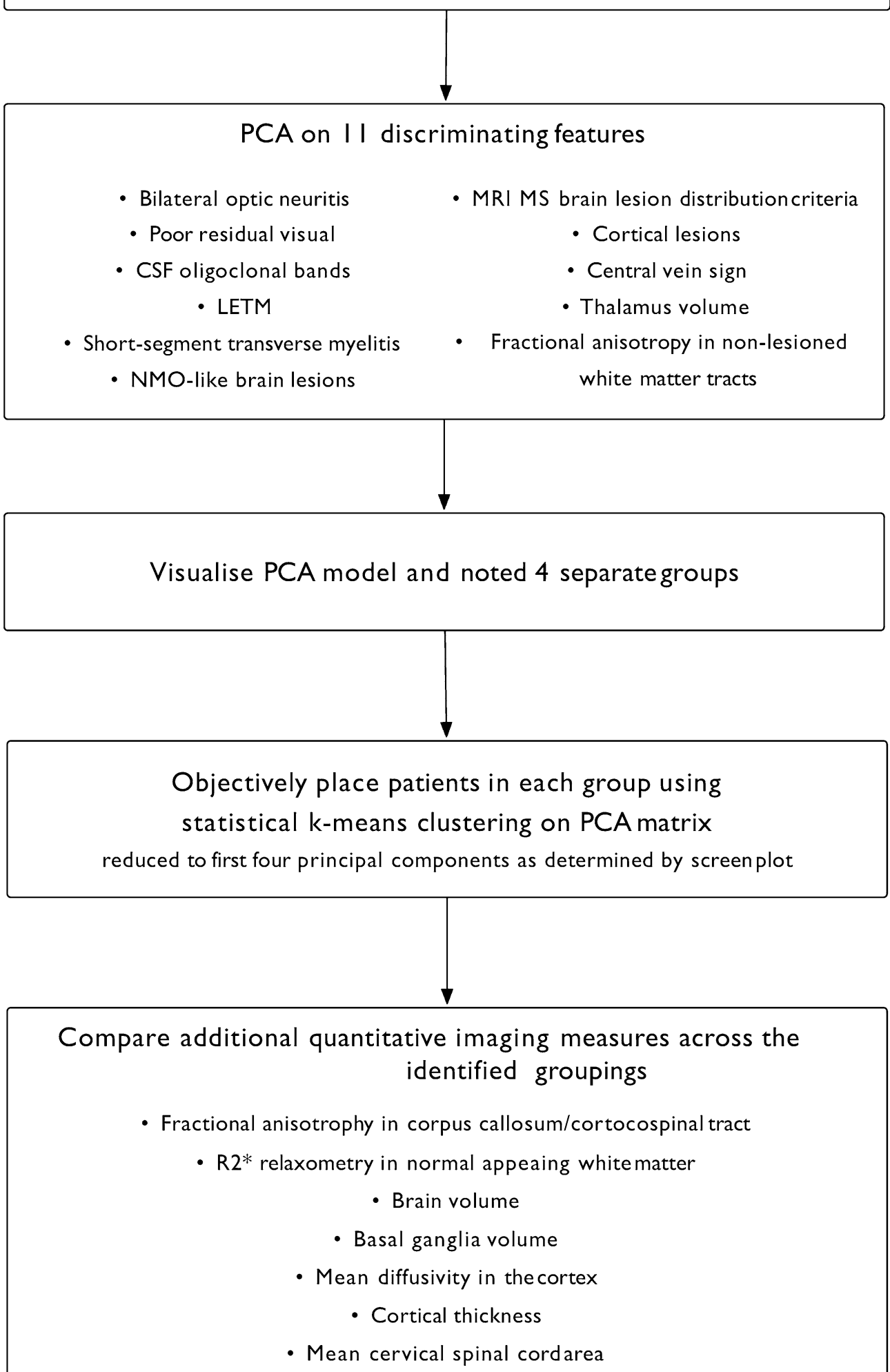

Fig. 1 The flow diagram presents the sequence of analysis steps allowing for the unsupervised identification of antibody-negative NMOSD/multiple sclerosis patient subgroupings followed by exploration of tissue damage-related quantitaive imaging measures witin the identified groups 


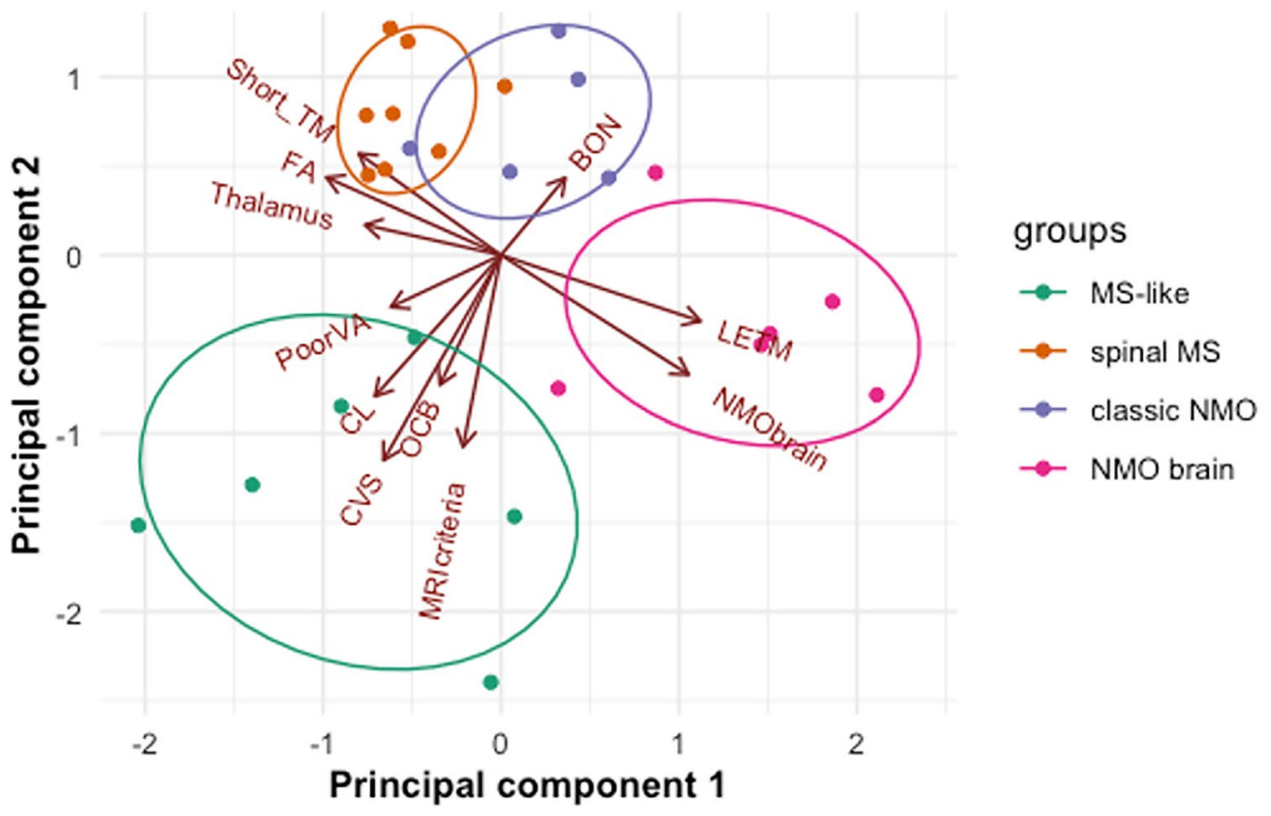

Fig. 2 Principal component analysis plot shows localisation of each individual patient (represented as a dot) according to their scoring on two first principal components. Ellipses and label colours represent patient subgroupings as identified by $k$-means clustering with four centres. The plot is overlaid with eigenvectors showing how each discriminating feature contributes to the location of the patient on the graph. The clusters are named for convenience depending on their predominating clinical and imaging features. $B O N$ bilateral optic neuritis, $C L$ cortical lesions, $C V S$ central vein sign, $F A$ fractional anisotropy in normal-appearing white matter tracts with the exclusion of optic radiation, LETM longitudinally extensive transverse myelitis, MRIcriteria MRI multiple sclerosis brain lesion distribution criteria, NMObrain neuromyelitis optica-like brain lesions, $O C B$ oligoclonal bands in the cerebrospinal fluid unmatched for serum, PoorVA residual visual acuity at $6 / 36$ or worse in at least one eye, Short_TM shortsegment transverse myelits, Thalamus thalamus volume

Table 1 Basic demographic, clinical information and breakdown of discriminating features in identified subgroups

\begin{tabular}{lllll}
\hline & $\begin{array}{l}\text { Group 1 } \\
\text { ("MS"-like) }\end{array}$ & $\begin{array}{l}\text { Group 2 } \\
\text { ("Spinal MS”-like) }\end{array}$ & $\begin{array}{l}\text { Group 3 } \\
\text { ("Classic NMO"-like) }\end{array}$ & $\begin{array}{l}\text { Group 4 } \\
\text { ("NMO-like" with } \\
\text { brain involvement) }\end{array}$ \\
\hline Number of patients & 6 & 8 & 5 & 6 \\
Female $\%$ & $17 \%$ & $63 \%$ & $80 \%$ & $83 \%$ \\
Mean age at scan (years, range) & $49(21-73)$ & $50(41-64)$ & $37(20-58)$ & $47(24-70)$ \\
Median disease duration (years, range) & $7(2-19)$ & $11(4-28)$ & $6(1-13)$ & $9(2-20)$ \\
Mean EDMUS (range) & $3(0-7)$ & $2.8(0-5)$ & $2.4(1-5)$ & $4(2-8)$ \\
Bilateral ON & $0 \%$ & $0 \%$ & $80 \%$ & $17 \%$ \\
Poor visual acuity & $33 \%$ & $13 \%$ & $40 \%$ & $17 \%$ \\
CSF OCB & $67 \%$ & $50 \%$ & $40 \%$ & $50 \%$ \\
LETM & $33 \%$ & $13 \%$ & $60 \%$ & $100 \%$ \\
Short-segment TM & $33.3 \%$ & $100 \%$ & $20 \%$ & $17 \%$ \\
NMO-like brain lesions & $17 \%$ & $0 \%$ & $0 \%$ & $67 \%$ \\
MRI brain criteria & $83 \%$ & $0 \%$ & $0 \%$ & $33 \%$ \\
Cortical lesions & $67 \%$ & $13 \%$ & $0 \%$ & $0 \%$ \\
Central vein sign & $83 \%$ & $0 \%$ & $0 \%$ & $0.49 \pm 0.01$ \\
FA & $0.49 \pm 0.01$ & $0.49 \pm 0.01$ & $1.0 \pm 0.05$ & $0.46 \pm 0.02 * *$ \\
Thalamus $\left(\mathrm{cm}{ }^{3}\right.$ ) & $0.98 \pm 0.13$ & $0.97 \pm 0.07$ & $0.84 \pm 0.12 *$ \\
\hline
\end{tabular}

These features were used to identify subgroups in the antibody-negative neuromyelitis optica/multiple sclerosis cohort using methods of unsupervised learning

The statistical significance of differences in non-conventional imaging measures across the subgroups is marked with asterisks: $* p<0.05$, $* * p<0.01$ 
in non-lesioned white matter tracts $(0.46 \pm 0.01$ vs. $0.49 \pm 0.01, p=0.003)$ and decreased thalamus volume $(0.84 \pm 0.12$ vs. $0.98 \pm 0.08, p=0.04)$. Table 1 shows basic demographic and clinical information on patients in each subgroup.

\section{Identified clusters correlate strongly with clinician's diagnosis}

Comparison with clinician's diagnosis revealed that likely MS was diagnosed only in patients from Group 1 and Group 2 (83\% and $88 \%$, respectively, Table 2), while likely NMOSD was diagnosed only in Group 3 and 4 (80\% and $83 \%$, respectively). Taking into account the breakdown of discriminating features and diagnoses for convenience we have termed Group 1 "MS-like", Group 2 "spinal MS-like", Group 3 "classic NMO"-like and Group 4 "NMO-like with brain involvement".

\section{Quantitative imaging differences between the identified groups in tissue damage parameters not used for subgroup identification}

Table 3 shows non-conventional imaging differences between four identified subgroups in parameters representing various aspects of disease pathology: normal-appearing white matter damage (fractional anisotropy in distinct white matter tracts, $\mathrm{R} 2 *$ relaxometry), axonal damage (normalised brain and subcortical structure volumes), cortical damage (mean diffusivity in the cortex, cortical thickness) and spinal cord damage (mean cervical spinal cord area).

\section{Group 1 and Group 2}

'MS-like' and 'spinal MS-like' patients did not differ significantly between each other in terms of normal-appearing white matter tract integrity (Fig. 3A, 3B) or atrophy measures in the brain but the latter group had a lower mean crosssectional area in the cervical spinal cord $(57.7 \pm 6.8$ vs. $61.4 \pm 4.3$, non-significant, Fig. 3C). Both groups combined
Table 2 Comparison between subgroups identified by unsupervised machine learning and clinician's diagnosis

\begin{tabular}{|c|c|c|c|c|}
\hline & $\begin{array}{l}\text { Group } 1 \\
\text { ("MS"-like) }\end{array}$ & $\begin{array}{l}\text { Group } 2 \\
\text { ("Spinal MS"- } \\
\text { like) }\end{array}$ & $\begin{array}{l}\text { Group } 3 \\
\text { (“Classic NMO"- } \\
\text { like) }\end{array}$ & $\begin{array}{l}\text { Group } 4 \\
\text { ("NMO-like" with } \\
\text { brain involvement) }\end{array}$ \\
\hline Number of patients & 6 & 8 & 5 & 6 \\
\hline MS diagnosis & $83 \%$ & $88 \%$ & $0 \%$ & $0 \%$ \\
\hline NMO diagnosis & $0 \%$ & $0 \%$ & $80 \%$ & $83 \%$ \\
\hline Other/undetermined & $17 \%$ & $12 \%$ & $20 \%$ & $17 \%$ \\
\hline
\end{tabular}

Table 3 Non-conventional magnetic resonance imaging measures in identified subgroups

\begin{tabular}{|c|c|c|c|c|}
\hline & $\begin{array}{l}\text { Group } 1 \\
\text { (“MS"-like) }\end{array}$ & $\begin{array}{l}\text { Group } 2 \\
\text { (“Spinal MS"-like) }\end{array}$ & $\begin{array}{l}\text { Group } 3 \\
\text { (“Classic NMO”-like) }\end{array}$ & $\begin{array}{l}\text { Group } 4 \\
\text { ("NMO-like" with } \\
\text { brain involvement) }\end{array}$ \\
\hline Fractional anisotropy in corpus callosum & $0.56 \pm 0.02$ & $0.58 \pm 0.02$ & $0.59 \pm 0.02$ & $0.48 \pm 0.04 * * *$ \\
\hline Fractional anisotropy in corticospinal tracts & $0.44 \pm 0.02$ & $0.44 \pm 0.01$ & $0.43 \pm 0.01$ & $0.43 \pm 0.01$ \\
\hline Fractional anisotropy in optic radiation & $0.52 \pm 0.03$ & $0.55 \pm 0.02$ & $0.54 \pm 0.01$ & $0.51 \pm 0.05$ \\
\hline $\begin{array}{l}\text { Mean R2* relaxometry in the normal-appearing } \\
\text { white matter }\end{array}$ & $21.2 \pm 0.58$ & $20.9 \pm 1.0$ & $21.5 \pm 1.0$ & $20.8 \pm 0.7$ \\
\hline Mean R2* relaxometry in the basal ganglia & $29.5 \pm 5.1$ & $28.9 \pm 2.9$ & $28 \pm 2.8$ & $27.9 \pm 4.2$ \\
\hline Normalised brain volume (1) & $1.48 \pm 0.14$ & $1.48 \pm 0.1$ & $1.50 \pm 0.09$ & $1.36 \pm 0.08$ \\
\hline Normalised basal ganglia volume $\left(\mathrm{cm}^{3}\right)$ & $13.3 \pm 1.6$ & $13.4 \pm 1.2$ & $12.3 \pm 1.6$ & $11.4 \pm 2.2$ \\
\hline Mean diffusivity in the cortex & $0.87 \pm 0.02$ & $0.87 \pm 0.03$ & $0.86 \pm 0.04$ & $0.92 \pm 0.03 *$ \\
\hline Mean cortical thickness & $2.74 \pm 0.1$ & $2.70 \pm 0.07$ & $2.77 \pm 0.13$ & $2.66 \pm 0.06$ \\
\hline Mean cervical spinal cord area & $61.4 \pm 4.3$ & $57.7 \pm 6.8$ & $65.7 \pm 5.1$ & $53.1 \pm 6.5^{*}$ \\
\hline
\end{tabular}

These measures were not used for subgroup identification

Statisitcally significant differences are marked with stars in the last column

$* p<0.05$

$* * p<0.01$

*** $p<0.001$ 
A

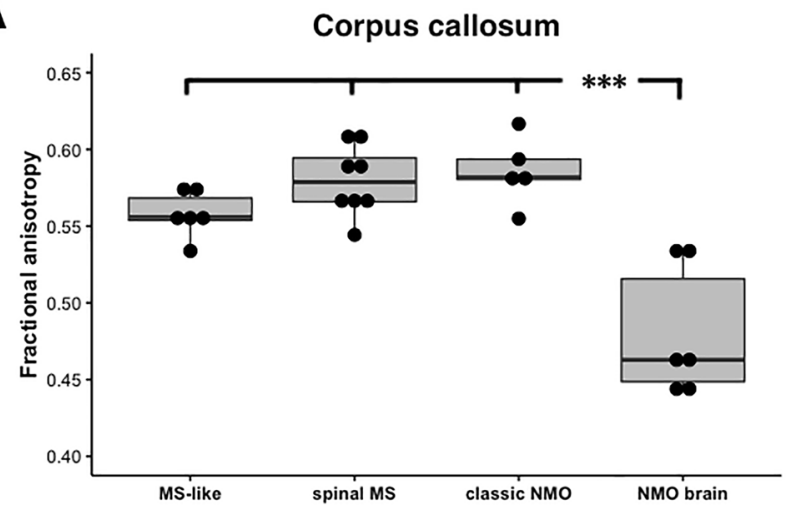

B

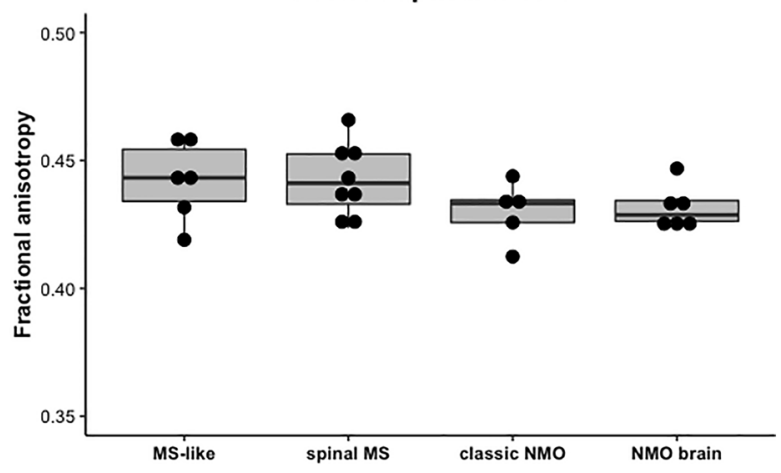

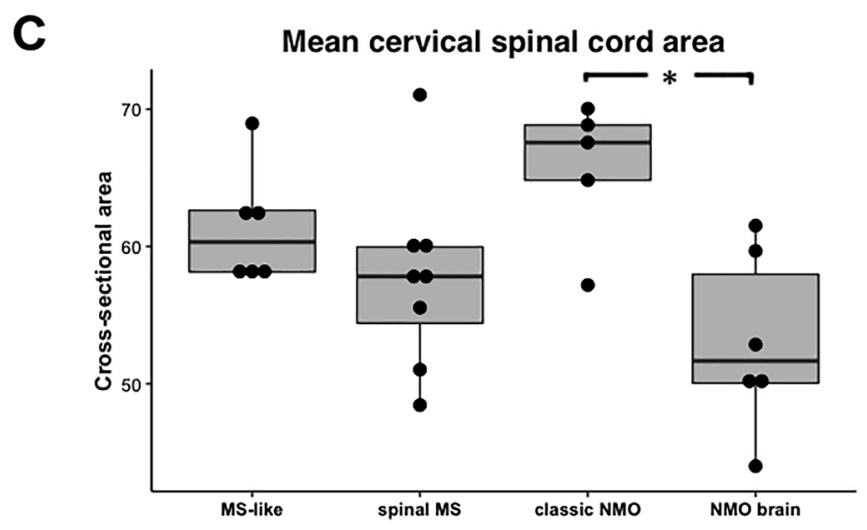

Fig. 3 Fractional anisotropy in the corpus callosum (A) and corticospinal tracts (B) in each identified subgroup. Group 4 shows significantly lower fractional anisotropy in the corpus callosum as compared with other groups $(* * * p<0.001)$, but no between-group difference is observed in corticospinal tracts. (C) Mean spinal cord cross-sectional area averaged across all eight cervical segments in four identified subgroups. Group 4 had significantly more atrophy than Group $3(* p=0.01)$. Statistically significant differences are marked with asterisks

\section{Group 4}

Given that low fractional anisotropy in normal-appearing white matter tracts (with the exclusion of the optic radiation) strongly contributed to the generation of "NMO-like with brain involvement" subgroup we analysed whether this difference is attributed to any particular white matter tract. Interestingly, we have found significant differences in fractional anisotropy in the corpus callosum when comparing between Group 4 and each of the three other groups ( $p<0.001$, Tukey's test), but not in corticospinal tracts (Fig. 3, Table 3). Cerebral volumetric measures (total brain, basal ganglia, brainstem volume, cortical thickness) were generally lower in this group as compared to other groups but this was not statistically significant (Table 3). Importantly, patients from this group had significantly higher mean diffusivity in the cortex as compared with "classic NMO-like" and "spinal MS-like" patients (Fig. 4). high cortical thickness and absence of cortical lesions, which all suggested absence of cortical pathology in this group (Fig. 4). 

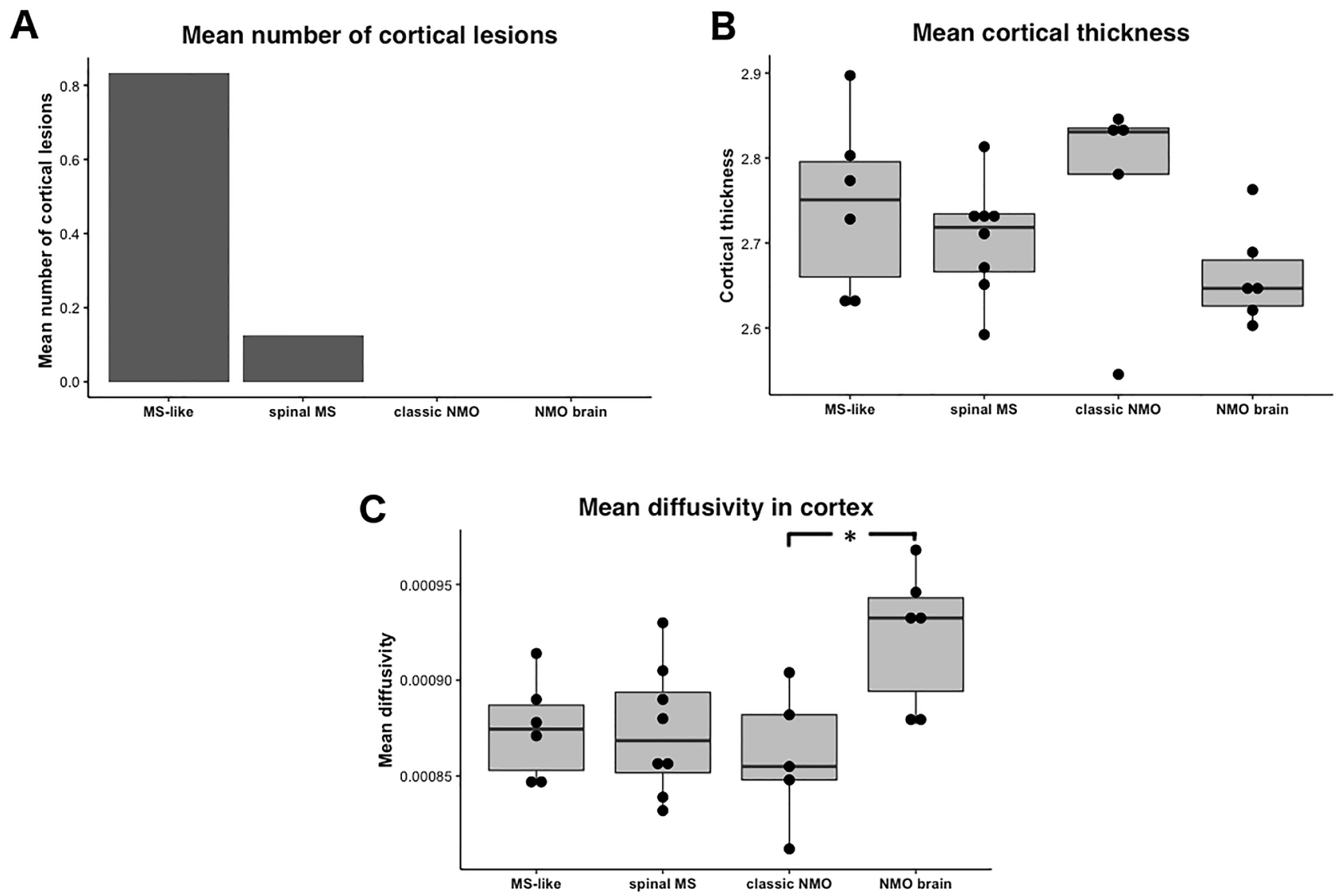

Fig. 4 Different aspects of cortical pathology are shown across four groups (A) mean number of cortical lesions, (B) mean cortical thickness, (C) mean diffusivity in the cortex. Mean diffusivity in the cor-

\section{Discussion}

We present the results of a prospective cross-sectional study of 25 double antibody-negative relapsing patients with NMOSD features and uncertain diagnosis. The aim of the study was to group the patients in an unsupervised, unbiased way into pathologically and/or clinically distinct subgroups on the basis of MS/NMOSD discriminatory features. We have found four separate groups existing in the cohort - two with predominantly MS-like features - in the brain (Group 1) or in the spinal cord (Group 2), one with optico-spinal "classic NMO-like" presentation (Group 3) and one with predominantly spinal cord and brain NMOSD-like disease (Group 4). We examined differences in non-conventional imaging findings. which were not used for subgroup identification, and found that while there were no significant differences between Groups 1 and 2, Group 4 displayed significantly more damage in the brain and spinal cord as compared with Group 3.

Current knowledge of antibody-negative NMOSD and the borderland between MS and NMOSD is very limited, as this group of patients has been largely neglected from previous tex was significantly higher in Group 4 as compared with Group 3 $(* p=0.02$, marked with an asterisk)

studies, most likely due to difficulties in the analysis resulting from diagnostic uncertainty. It is understood that this group might include atypical MS, NMOSD mediated by yet undiscovered antibodies and other conditions such as neurosarcoid. The condition can be disabling in its own right and more so if treated inappropriately [28]. Most expert clinicians would opt for global immunosuppression rather than MS-modifying drugs in case of diagnostic doubt [10] but it is unclear how effective this approach is in terms of preventing disability. Interestingly, antibody-negative NMOSD appears to respond differently to treatment as compared with aquaporin-4-antibody-positive NMOSD. In recent clinical trials neither satralizumab, anti-IL-6 antibody, nor inebilizumab, anti-CD19 drug, showed any signal of efficacy in antibody-negative NMOSD [29-31]. The latter finding was particularly interesting as B cell-depleting therapy is often considered by clinicians to be the most appropriate for patients with overlapping symptoms of NMOSD and MS. All this points to a compelling need to classify the antibodynegative cases to help choose appropriate treatment.

There are several limitations of the study. These include the supervised choice of discriminators, which might bias 
the generation of clusters. To reduce this bias we included only the most typical NMOSD and MS features (see "Methods") combined with well-established or promising nonconventional imaging discriminators (cortical lesions [32], central vein sign [33], thalamus volume, fractional anisotropy in normal-appearing white matter [34]). In the analysis multiple imaging outcomes were used for a small number of patients however most non-conventional measures were assessed after the subgroups have been identified to explore if there might be differences in the underlying tissue damage patterns/pathologies between the groups. The small number of patients is due to the relative rarity of antibody-negative NMOSD patients [35] and their frequent labelling as atypical MS. Of note, we focussed only on relapsing patients who definitely have a chronic disease and excluded monophasic illness, for example viral or post-viral, which typically would not be considered for long-term disease-modifying treatment. Although rare, relapsing antibody-negative NMOSD patients cause the most difficulty in the clinic in terms of diagnosis and thus treatment decisions [10]. Another weakness is the lack of a gold standard reference for the ultimate diagnosis, which is also the reason to do this study and why the literature in this area is sparse.

Patients diagnosed with likely antibody-negative NMO in the clinic were all assigned to Group 3 or 4 by the unsupervised algorithm, and these groups differed significantly between each other. Patients from Group 4 had highly destructive disease of the spinal cord and brain as seen in advanced MS, however, they all had a number of red flags for the diagnosis of MS as well. First, all these patients had a history of LETM, considered a hallmark of NMOSD [36] and extremely rare in MS [37]. Four out of six had NMO-like brain lesions, including a lesion adjacent to the 3 rd ventricle (patient 3), periaqueductal and hypothalamic lesions (patient 13), oedematous lesion involving the complete thickness of the splenium of corpus callosum (patient 20) and a large hemispheric white matter lesion (patient 21). One patient in this group had a dramatic exacerbation on natalizumab with a formation of ring-enhancing brain lesions (patient 12). These patients are likely to have a disease process that is different from MS, potentially mediated by autoantibodies or other type of inflammation. One of these patients underwent biopsy of the cerebral lesion (Patient 21) which showed chronic inflammation and reactive gliosis without evidence of MS demyelination, granulomatous process, vasculitis or neoplasm. This group may represent a new disease entity.

All patients from Group 3 had a normal brain appearance based on standard imaging and significantly less brain atrophy or white matter disintegrity when compared with Group 4. These patients had predominantly optico-spinal presentation but they had less spinal cord atrophy than Group 4 despite a high proportion of previous LETM in both groups. Two out of five had attacks of both BON and LETM, two had recurrent isolated optic neuritis including at least one attack of simultaneous BON and one had LETM followed several months later by unilateral optic neuritis. Two out of five in this group were left with poor residual visual acuity. This group, as opposed to other groups, did not have any evidence of cortical damage, was the least disabled of all groups (average EDMUS 2.4) and appeared to have a milder form of NMOSD with a high proportion of BON, similar to what is observed in MOGAD [13]. We find it likely that disease process in this group is mediated by yet undiscovered antibodies.

Patients from Groups 1 and 2, despite being referred to NMO clinic, appear to have forms of MS. Brain lesions in these patients, although there were typically only a few of them, were positive for landmark MS features (Group 1). Those without brain lesions (Group 2) had short-segment lesions in the spinal cord but very rarely had NMOSD features, such as LETM $(12.5 \%)$, BON $(0 \%)$, poor visual recovery $(12.5 \%)$ or NMO brain lesions $(0 \%)$. Of the patients in this group, $50 \%$ had unmatched oligoclonal bands in the CSF. This form of spinal MS may turn out to be pathologically distinct and is worth further research.

In conclusion, to our knowledge this is the first study focussing on the heterogenous cohort of antibody-negative NMO/MS patients using non-conventional imaging and unsupervised unbiased clustering algorithms to subclassify these patients and guide treatment decisions. Our results suggest that differential diagnosis in NMOSD/MS patients might benefit from the use of novel imaging techniques not yet widely used in clinical practice, including central vein sign, cortical lesions and the implementation of MS MRI brain lesion distribution criteria. If present these biomarkers might direct clinicians towards the likely diagnosis of MS. We suspect patients who are negative for these features and have a normal brain imaging might still have the diagnosis of MS if they present with spinal short-segment disease either isolated or associated with unilateral optic neuritis. We also believe that patients with a history of LETM have a non-MS disease. Those with LETM who have a history of BON might have NMOSD mediated by yet undiscovered antibodies, while those with MS-atypical brain lesions and significant brain atrophy could possibly have a diagnosis alternative to MS or 'true' NMOSD. Further prospective studies in identified subgroups, correlated with clinico-pathological studies and antibody research, will help clarify the underlying processes in each group.

Supplementary Information The online version contains supplementary material available at https://doi.org/10.1007/s00415-021-10619-1.

Acknowledgements Dr Juryńczyk and co-authors thank all the patients who kindly agreed to participate in the study and took the time and effort to come to Oxford and undergo the research scan in Wellcome Centre for Integrative Neuroimaging. Dr Juryńczyk and Dr 
Klimiec-Moskal thank Dr Romina Mariano (Specialist NMO Clinic, John Radcliffe Hospital, Oxford) for insights into the analysis of the spinal cord images.

Funding The study was funded by Oxford University Hospitals Research and Development grant A20069. This publication also arises from research funded by the John Fell Oxford University Press (OUP) Research Fund. We gratefully acknowledge the Highly Specialised Commissioning Team for funding the NMO service in Oxford. Dr Juryńczyk received research fellowship from the Polish Ministry of Science and Higher Education programme Mobilnosc Plus (1070/ $\mathrm{MOB} / \mathrm{B} / 2013 / 0$ ) and funding from NAWA Polish Returns programme PPN/PPO/2020/1/00043/U/00001. Dr Klimiec-Moskal received Newsom-Davis fellowship from the Guarantors of Brain. Prof. Jenkinson is supported by the National Institute for Health Research (NIHR) Oxford Biomedical Research Centre (BRC), and the Wellcome Centre for Integrative Neuroimaging, which is supported by core funding from the Wellcome Trust (203139/Z/16/Z).

Data availability All data and material are available from the corresponding authors upon request.

Code availability Custom codes are available from the corresponding authors upon request.

\section{Declarations}

Conflicts of interest Prof. Jenkinson receives royalties from licensing of FSL to non-academic, commercial parties. The remaining authors report no competing interests.

Ethics approval The study was approved by The National Institute for Social Care and Health Research Ethics Committee (14/WA/1252).

Consent to participate All participants signed written informed consent to participate in the study.

Consent for publication All participants signed written informed consent for the publication of study results.

Open Access This article is licensed under a Creative Commons Attribution 4.0 International License, which permits use, sharing, adaptation, distribution and reproduction in any medium or format, as long as you give appropriate credit to the original author(s) and the source, provide a link to the Creative Commons licence, and indicate if changes were made. The images or other third party material in this article are included in the article's Creative Commons licence, unless indicated otherwise in a credit line to the material. If material is not included in the article's Creative Commons licence and your intended use is not permitted by statutory regulation or exceeds the permitted use, you will need to obtain permission directly from the copyright holder. To view a copy of this licence, visit http://creativecommons.org/licenses/by/4.0/.

\section{References}

1. Wingerchuk DM, Hogancamp WF, O'Brien PC, Weinshenker BG (1999) The clinical course of neuromyelitis optica (Devic's syndrome). Neurology 53:1107-1107. https://doi.org/10.1212/WNL. 53.5.1107
2. Lennon VA, Wingerchuk DM, Kryzer TJ et al (2004) A serum autoantibody marker of neuromyelitis optica: distinction from multiple sclerosis. Lancet 364:2106-2112

3. Lennon VA, Kryzer TJ, Pittock SJ et al (2005) IgG marker of optic-spinal multiple sclerosis binds to the aquaporin-4 water channel. J Exp Med 202:473-477. https://doi.org/10.1084/jem. 20050304

4. Wingerchuk DM, Lennon VA, Lucchinetti CF et al (2007) The spectrum of neuromyelitis optica. Lancet Neurol 6:805-815. https://doi.org/10.1016/S1474-4422(07)70216-8

5. Mader S, Gredler V, Schanda K et al (2011) Complement activating antibodies to myelin oligodendrocyte glycoprotein in neuromyelitis optica and related disorders. J Neuroinflammation 8:184. https://doi.org/10.1186/1742-2094-8-184

6. Kitley J, Woodhall M, Waters P et al (2012) Myelin-oligodendrocyte glycoprotein antibodies in adults with a neuromyelitis optica phenotype. Neurology 79:1273-1277. https://doi.org/10. 1212/WNL.0b013e31826aac4e

7. Jurynczyk M, Geraldes R, Probert F et al (2017) Distinct brain imaging characteristics of autoantibody-mediated CNS conditions and multiple sclerosis. Brain 140:617-627. https://doi.org/ 10.1093/brain/aww350

8. Matthews L, Marasco R, Jenkinson M et al (2013) Distinction of seropositive NMO spectrum disorder and MS brain lesion distribution. Neurology 81:1966. https://doi.org/10.1212/01. wnl.0000436079.95856.1f

9. Juryńczyk M, Tackley G, Kong Y et al (2017) Brain lesion distribution criteria distinguish MS from AQP4-antibody NMOSD and MOG-antibody disease. J Neurol Neurosurg Psychiatry. https://doi.org/10.1136/jnnp-2016-314005

10. Juryńczyk M, Weinshenker B, Akman-Demir G et al (2015) Status of diagnostic approaches to AQP4-IgG seronegative NMO and NMO/MS overlap syndromes. J Neurol 263:140-149. https://doi.org/10.1007/s00415-015-7952-8

11. Thompson AJ, Banwell BL, Barkhof F et al (2018) Diagnosis of multiple sclerosis: 2017 revisions of the McDonald criteria. Lancet Neurol 17:162-173. https://doi.org/10.1016/S14744422(17)30470-2

12. Jarius S, Ruprecht K, Kleiter I et al (2016) MOG-IgG in NMO and related disorders: a multicenter study of 50 patients. Part 2: epidemiology, clinical presentation, radiological and laboratory features, treatment responses, and long-term outcome. J Neuroinflamm. https://doi.org/10.1186/s12974-016-0718-0

13. Jurynczyk M, Messina S, Woodhall MR et al (2017) Clinical presentation and prognosis in MOG-antibody disease: a UK study. Brain 140:3128-3138. https://doi.org/10.1093/brain/ awx 276

14. Cacciaguerra L, Meani A, Mesaros S et al (2019) Brain and cord imaging features in neuromyelitis optica spectrum disorders. Ann Neurol 85:371-384. https://doi.org/10.1002/ana.25411

15. Wingerchuk DM, Banwell B, Bennett JL et al (2015) International consensus diagnostic criteria for neuromyelitis optica spectrum disorders. Neurology 85:177-189. https://doi.org/10.1212/WNL. 0000000000001729

16. Kleiter I, Hellwig K, Berthele A et al (2012) Failure of natalizumab to prevent relapses in neuromyelitis optica. Arch Neurol 69:239-245. https://doi.org/10.1001/archneurol.2011.216

17. Palace J, Leite MI, Nairne A, Vincent A (2010) Interferon Beta treatment in neuromyelitis optica: increase in relapses and aquaporin 4 antibody titers. Arch Neurol 67:1016-1017. https://doi. org/10.1001/archneurol.2010.188

18. Sati P, Oh J, Todd Constable R et al (2016) The central vein sign and its clinical evaluation for the diagnosis of multiple sclerosis: a consensus statement from the North American imaging in multiple sclerosis cooperative. Nat Rev Neurol 12:714-722. https:// doi.org/10.1038/nrneurol.2016.166 
19. Smith SM, Zhang Y, Jenkinson M et al (2002) Accurate, robust, and automated longitudinal and cross-sectional brain change analysis. Neuroimage 17:479-489. https://doi.org/10.1006/nimg.2002. 1040

20. Patenaude B, Smith SM, Kennedy DN, Jenkinson M (2011) A Bayesian model of shape and appearance for subcortical brain segmentation. Neuroimage 56:907-922. https://doi.org/10.1016/j. neuroimage.2011.02.046

21. Dale AM, Fischl B, Sereno MI (1999) Cortical surface-based analysis: I segmentation and surface reconstruction. Neuroimage 9:179-194. https://doi.org/10.1006/nimg.1998.0395

22. Wakana S, Jiang H, Nagae-Poetscher LM et al (2004) Fiber tractbased atlas of human white matter anatomy. Radiology 230:7787. https://doi.org/10.1148/radiol.2301021640

23. De Leener B, Lévy S, Dupont SM et al (2017) SCT: spinal cord toolbox, an open-source software for processing spinal cord MRI data. Neuroimage 145:24-43. https://doi.org/10.1016/j.neuro image.2016.10.009

24. Filippi M, Cercignani M, Inglese M et al (2001) Diffusion tensor magnetic resonance imaging in multiple sclerosis. Neurology 56:304-311. https://doi.org/10.1212/WNL.56.3.304

25. Ciccarelli O, Werring DJ, Wheeler-Kingshott CAM et al (2001) Investigation of MS normal-appearing brain using diffusion tensor MRI with clinical correlations. Neurology 56:926-933. https:// doi.org/10.1212/WNL.56.7.926

26. Cercignani M, Inglese M, Pagani E et al (2001) Mean diffusivity and fractional anisotropy histograms of patients with multiple sclerosis. Am J Neuroradiol 22:952-958

27. Tallantyre EC, Dixon JE, Donaldson I et al (2011) Ultra-high-field imaging distinguishes MS lesions from asymptomatic white matter lesions. Neurology 76:534-539. https://doi.org/10.1212/WNL. 0b013e31820b7630

28. Kitley J, Evangelou N, Küker W et al (2014) Catastrophic brain relapse in seronegative NMO after a single dose of natalizumab. J Neurol Sci. https://doi.org/10.1016/j.jns.2014.01.035

29. Yamamura T, Kleiter I, Fujihara K et al (2019) Trial of satralizumab in neuromyelitis optica spectrum disorder. N Engl J Med 381:2114-2124. https://doi.org/10.1056/NEJMoa1901747
30. Traboulsee A, Greenberg BM, Bennett JL et al (2020) Safety and efficacy of satralizumab monotherapy in neuromyelitis optica spectrum disorder: a randomised, double-blind, multicentre, placebo-controlled phase 3 trial. Lancet Neurol 19:402-412. https:// doi.org/10.1016/S1474-4422(20)30078-8

31. Cree BAC, Bennett JL, Kim HJ et al (2019) Inebilizumab for the treatment of neuromyelitis optica spectrum disorder (N-MOmentum): a double-blind, randomised placebo-controlled phase $2 / 3$ trial. Lancet 394:1352-1363. https://doi.org/10.1016/S01406736(19)31817-3

32. Calabrese M, Oh MS, Favaretto A et al (2012) No MRI evidence of cortical lesions in neuromyelitis optica. Neurology 79:16711676. https://doi.org/10.1212/WNL.0b013e31826e9a96

33. Cortese R, Magnollay L, Tur C et al (2018) Value of the central vein sign at 3T to differentiate MS from seropositive NMOSD. Neurology 90:e1183-e1190. https://doi.org/10.1212/WNL.00000 00000005256

34. Kremer S, Renard F, Achard S et al (2015) Use of advanced magnetic resonance imaging techniques in neuromyelitis optica spectrum disorder. JAMA Neurol 72:815. https://doi.org/10.1001/ jamaneurol.2015.0248

35. O'Connell K, Hamilton-Shield A, Woodhall M et al (2020) Prevalence and incidence of neuromyelitis optica spectrum disorder, aquaporin-4 antibody-positive NMOSD and MOG antibody-positive disease in Oxfordshire, UK. J Neurol Neurosurg Psychiatry 91:1126-1128. https://doi.org/10.1136/jnnp-2020-323158

36. Wingerchuk D, Banwell B, Bennett J et al (2014) Revised diagnostic criteria for neuromyeltiis optica spectrum disorders. Neurology 82:S63.001

37. Asnafi S, Morris PP, Sechi E et al (2020) The frequency of longitudinally extensive transverse myelitis in MS: a populationbased study. Mult Scler Relat Disord 37:101487. https://doi.org/ 10.1016/j.msard.2019.101487 